\title{
Effectiveness of low-level laser therapy and chewing gum in reducing orthodontic pain: A randomized controlled trial
}

\author{
Fatih Celebi (1) \\ Ali Altug Bicakci \\ Ufuk Kelesoglu
}

Department of Orthodontics, Faculty of Dentistry, Tokat Gaziosmanpasa University, Tokat, Turkey

\begin{abstract}
Objective: The purpose of this study was to evaluate the effects of chewing gum and low-level laser therapy in alleviating orthodontic pain induced by the initial archwire. Methods: Patients with 3-6 mm maxillary crowding who planned to receive non-extraction orthodontic treatment were recruited for the study. Sixty-three participants ( 33 females and 30 males) were randomly allocated into three groups: laser, chewing gum, and control. In the laser group, a gallium aluminum arsenide (GaAlAs) diode laser with a wavelength of $820 \mathrm{~nm}$ was used to apply a single dose immediately after orthodontic treatment began. In the chewing gum group, sugar-free gum was chewed three times for 20 minutesimmediately after starting treatment, and at the twenty-fourth and forty-eighth hours of treatment. Pain perception was measured using a visual analog scale at the second, sixth, and twenty-fourth hours, and on the second, third, and seventh days. Results: There were no statistically significant differences between the groups at any measured time point $(p>0.05)$. The highest pain scores were detected at the twenty-fourth hour of treatment in all groups. Conclusions: Within the limitations of the study, we could not detect whether low-level laser therapy and chewing gum had any clinically significant effect on orthodontic pain. Different results may be obtained with a higher number of participants or using lasers with different wavelengths and specifications. Although the study had a sufficient number of participants according to statistical analysis, higher number of participants could have provided more definitive outcomes.
\end{abstract}

[Korean J Orthod 2021;51(5):313-320]

Key words: Chewing gum, Low-level laser therapy, Pain

Received October 20, 2020; Revised January 11, 2021; Accepted January 29, 2021.

Corresponding author: Fatih Celebi.

Associate Professor, Department of Orthodontics, Faculty of Dentistry, Tokat Gaziosmanpasa University, Kaleardı Mahallesi Muhittin Fisunoğlu Caddesi Omcalık Sokak Ali Şevki EREK Yerleşkesi, Merkez/Tokat 60100, Turkey.

Tel +90-555-604-21-55 e-mail fatihcelebi5860@gmail.com

How to cite this article: Celebi F, Bicakci AA, Kelesoglu U. Effectiveness of low-level laser therapy and chewing gum in reducing orthodontic pain: A randomized controlled trial. Korean J Orthod 2021;51:313-320.

(C) 2021 The Korean Association of Orthodontists.

This is an Open Access article distributed under the terms of the Creative Commons Attribution Non-Commercial License (http://creativecommons.org/licenses/by-nc/4.0) which permits unrestricted non-commercial use, distribution, and reproduction in any medium, provided the original work is properly cited. 


\section{INTRODUCTION}

Pain is one of the most common side effects of orthodontic treatment. Particularly, archwire activation, separator-band placement, debonding, and mini-screw applications cause pain and discomfort. ${ }^{1-3}$ Although the percentage varies according to different studies, on average, it has been reported that between $80-95 \%$ of patients receiving orthodontic treatment experienced pain. ${ }^{1,2,4,5}$ It was ranked fourth among the fears related to orthodontic treatment, and first among the most disliked experiences during treatment. ${ }^{6}$ Additionally, anxiety regarding pain negatively impacts oral hygiene. Many patients prefer a softer diet, believing it to cause less pain; however, soft and sticky food may increase the risk of plaque formation and contribute to the deterioration of oral hygiene. Those who experience pain may also avoid using a toothbrush so as not to exacerbate the problem.

The pressure pain threshold is described as the minimum applied force that causes pain. ${ }^{7}$ It is measured using a device called algometer or dolorimeter. The measurement is carried out by applying pressure to the subject's skin at a constant rate via a small rubber footplate. The least amount of pressure that causes pain is recorded and called the pressure pain threshold. It was developed in the 1930s and has been widely used in clinical practice, especially in physical medicine and rehabilitation, since the $1980 \mathrm{~s}^{8}{ }^{8}$

This method documents the pain threshold by measuring the tenderness levels in muscles, joints, tendons, and ligaments. Teeth are surrounded by the periodontal ligament within the alveolar bone. The pain process during orthodontic tooth movement occurs within this ligament. Therefore, it is plausible that the pressure-based pain threshold measurement using the algometer can also be applied for orthodontic pain. The pain threshold has been mentioned as one of the individual factors that affect the perception of orthodontic pain. 'However, this factor has been ignored in orthodontic pain studies to date.

Low-level laser therapy (LLLT) is defined as a laser treatment that will not cause the body temperature to rise above $36.5^{\circ} \mathrm{C} .{ }^{9}$ It has been reported that LLLT has a biostimulation effect on fibroblastic activity, cartilage proliferation, collagen synthesis, nerve regeneration, and bone remodeling..$^{10,11}$ Most of the past investigations were based on its therapeutic effects in acceleration of wound healing, pain control, and reduction of inflammation. ${ }^{9,12,13}$ Studies have also been carried out on the use of LLLT in orthodontics with the aim of managing pain and accelerating tooth movement. ${ }^{9,14}$ There is no consensus regarding its pain-relief effect in literature, as studies have presented conflicting results. ${ }^{9,15,16}$
Chewing gum was presented as an alternative pain-relief method in orthodontics. ${ }^{17}$ It was believed that chewing gum displaced the teeth temporarily, loosening the compressed periodontal structures, including nerve fibers and occluded blood vessels, thus enabling the blood to flow more easily. This prevented the action of biochemical agents, such as algogenic substances, that could cause pain. In fact, other pain-relief methods studied in orthodontics, such as vibration and chewing wafers, were based on the same principle. However, studies have reported conflicting results regarding its pain-relief effect, with no consensus in the literature. ${ }^{17,18}$

The aim of this study was to investigate whether lowlevel lasers and chewing gum can reduce orthodontic pain.

\section{MATERIALS AND METHODS}

This study was reviewed and approved by the Clinical Research Ethics Committee of Tokat Gaziosmanpasa University (approval number:19-KAEK-120). The sample size was determined using the Power Analysis and Sample Size software (PASS 2008; NCSS, Kaysville, UT, USA). The minimum number of participants per group was calculated to be 19 , to achieve a power of $80 \%$ for a clinically significant difference. Sixty-three participants between the ages of 12 and 24 years, who had 3-6 mm of maxillary crowding, non-extraction treatment modality, permanent dentition, and healthy teeth and gums, were enrolled in the study. Exclusion criteria included the chronic use of analgesics, unerupted teeth, medical histories, and the use of transpalatal arches, miniscrews, or headgear as part of orthodontic treatment. Informed consent was obtained from all participants and their parents.

Participants were randomly allocated to three groups: low-level laser, chewing gum, and control, such that all groups included 11 female and 10 male participants. Randomization was performed using red and blue raffle boxes for female and male participants, respectively. Their pressure pain thresholds were measured using a dolorimeter. Measurements were made by applying pressure to the palm skin around the thumb muscles with the footplate of the device. Three measurements were taken from different locations for each patient and their average value was assigned as the pain threshold score. The reliability and reproducibility of this method have been shown in previous studies. ${ }^{19,20}$ It was planned to exclude participants if they disrupted the equal distribution across the groups, in terms of pain threshold, and to enroll new participants. The Kruskal-Wallis test was performed to check the equal distribution of the participants across the groups, in terms of pain threshold. In line with the guidance of statistical analysis, six patients 
who disrupted the equality among the groups were excluded from the study, and substituted with new patients. Consolidated standards of reporting trials (CONSORT) flow diagram of the study is presented in Figure 1.

Fixed orthodontic treatment was initiated in all participants with Roth prescription brackets and tubes of $0.018 \times$ 0.025-inch and 0.014-inch round nickel-titanium archwire. Archwire was engaged using elastic ties and cut distal to the first molar tubes, in such a way that they did not irritate the buccal mucosa. Treatment was initiated in the maxilla only; no application took place on the mandibular arch. The participants were instructed to maintain oral hygiene and were asked not to take any medication during the study.

In the laser group, LLLT was initiated immediately after the start of the fixed orthodontic treatment, and a single dose was applied. A gallium aluminum arsenide diode laser (CTL-1106MX; Doris, Warsaw, Poland) was used with a wavelength of $820 \mathrm{~nm}$ and with the technical specifications of a power output of $50 \mathrm{~mW}$, an energy dose of $0.8 \mathrm{~J}$, an exposure duration of 16 seconds, a focal spot area of $0.453 \mathrm{~cm}^{2}$, a power density of 110.3 $\mathrm{mW} / \mathrm{cm}^{2}$, and an energy density of $1.76 \mathrm{~J} / \mathrm{cm}^{2}$ (Figure 2). Laser irradiation was used on both the buccal and palatal mucosa, covering a total of 12 teeth, from the maxillary left first molar to the right first molar. A total of six regions, three on each side, were irradiated per tooth: the distal aspect of the apical third, the midsection of the middle third, and the mesial aspect of the cervical third (Figure 3). In the chewing gum group, sugarfree gum was chewed for 20 minutes, just after the orthodontic treatment was initiated. The procedure was repeated after 24 and 48 hours. Laser application and gum chewing sessions were both conducted at the clinic and under supervision. No intervention was performed in the control group, aside from routine orthodontic treatment.

A visual analog scale (VAS) based on a $10 \mathrm{~cm}$ ruler was used to measure pain perception. Measurements were performed at six time points during the treatment: the second hour, the sixth hour, the first day, the second day, the third day, and the seventh day. VAS diaries

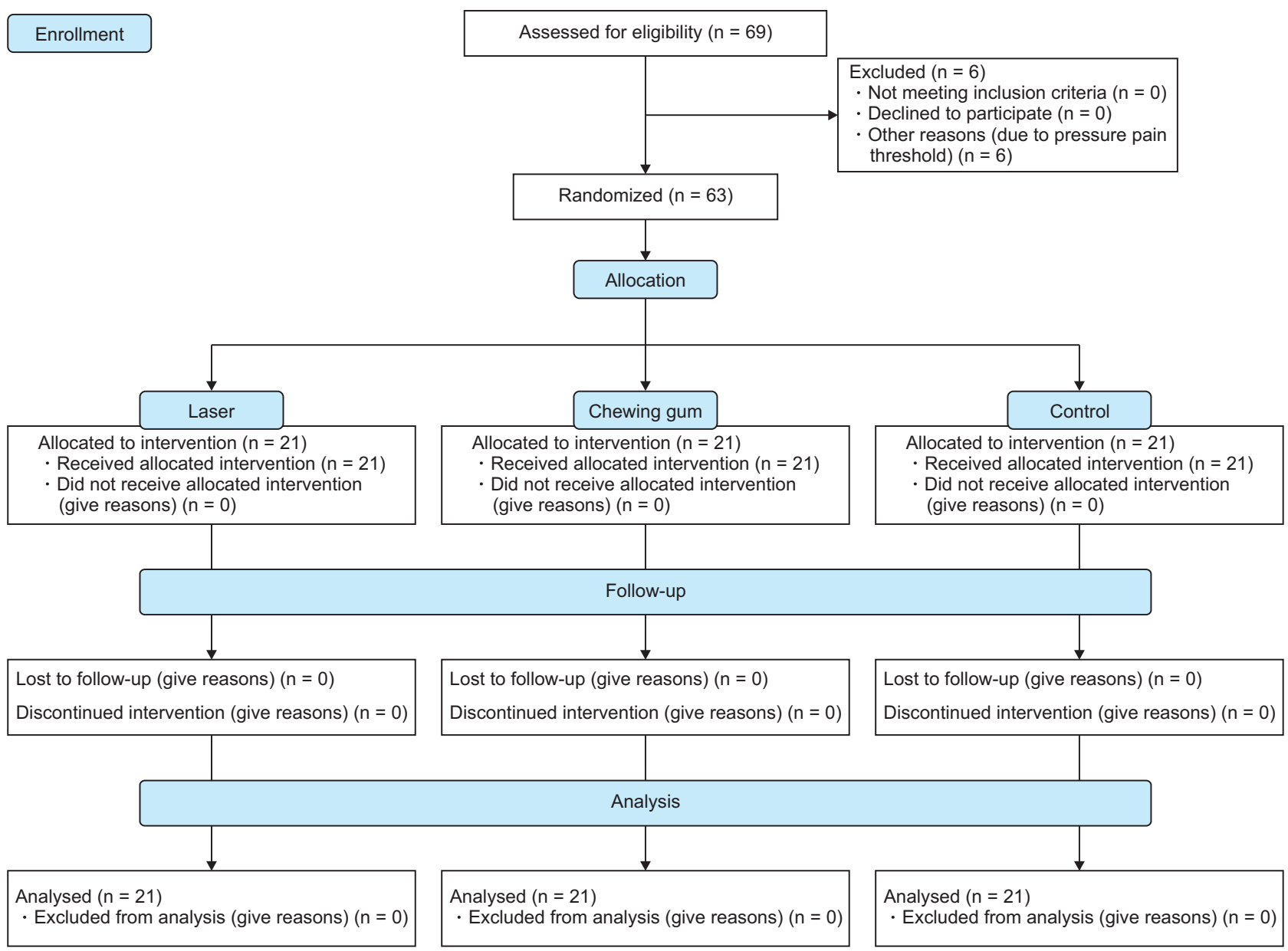

Figure 1. Consolidated standards of reporting trials (CONSORT) flow diagram of the study. 
containing six measurement sheets were prepared for each participant. Participants were instructed on how to fill in the sheets. To evoke their pain perception prior to measurement, participants were asked to tap their teeth ten times, by opening and closing their mouths, and to apply pressure on each tooth using their thumb.

\section{Statistical analysis}

Data were statistically analyzed using the SPSS version 25.0 (IBM Corp., Armonk, NY, USA). The ShapiroWilk test was utilized in evaluating the distribution of the data. The levene test was used to evaluate the homogeneity of the variance. For intergroup comparisons, one-way ANOVA and Kruskal-Wallis tests were used to evaluate age and algometer scores, respectively. The Kruskal-Wallis test was used to evaluate VAS scores among the groups. Statistical significance was considered for $p$-values less than 0.05 .

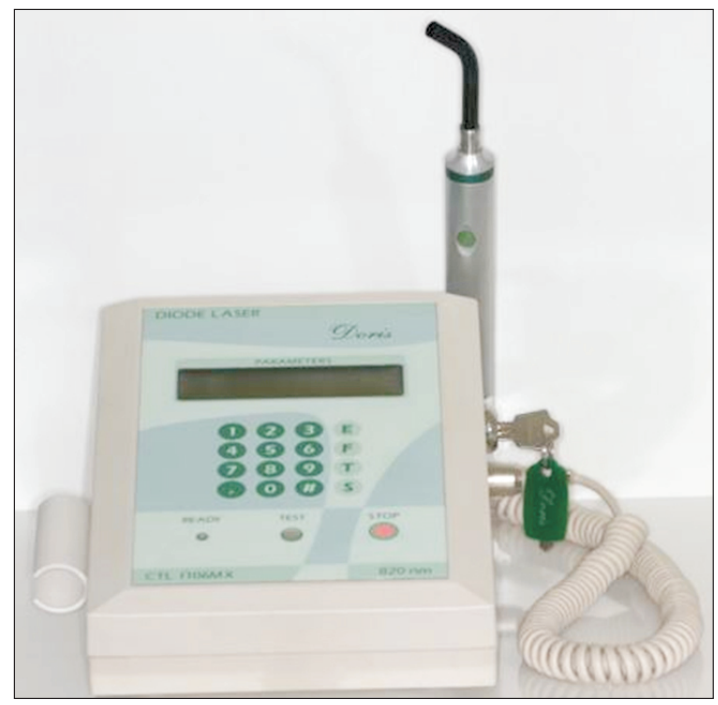

Figure 2. Laser device used in the study. A gallium aluminum arsenide diode laser (CTL-1106MX; Doris, Warsaw, Poland) with $820 \mathrm{~nm}$ wavelength was used.

\section{RESULTS}

There were no statistically significant differences among the groups in terms of age $(p=0.587)$ and pressure pain threshold scores $(p=0.057$, Table 1$)$. The mean ages of the LLLT, chewing gum, and control groups were 15.4 years, 15.8 years, and 15.3 years, respectively. The mean algometer scores of the LLLT, chewing gum, and control groups were 13.9, 17.4, and 15.3 respectively. Statistically significant differences were not detected between the groups in terms of VAS scores at any time point (second hour $=0.465$, sixth hour $=$ 0.610 , first day $=0.786$, second day $=0.833$, third day $=0.757$, seventh day $=0.774$; Table 2 ) .

The highest levels of pain in all groups were reached by the twenty-fourth hour of treatment. The general patterns of pain in all the groups were similar. The pain recorded at the second hour increased gradually and peaked at the end of the first day. It decreased gradually, without following a plateau curve after reaching the highest point. Pain levels were clinically insignificant by the seventh day (Figure 4).

\section{DISCUSSION}

The biggest challenge faced in pain related studies is that pain is a subjective phenomenon affected by individual variations. This has led to considerable inconsistencies in the results of the such investigations. Equality between groups, in terms of individual characteristics, is important in all researches. However, it is crucial in pain studies because many individual factors such as gender, age, pain threshold, and applied force can affect the perception of pain. ' In this study, individual variables among the groups were equalized as much as possible. An equal number of female and male participants were included in each group. The orthodontic force was equalized for each participant by using 0.014-inch $\mathrm{Ni}$ Ti archwire and by assigning 3-6 $\mathrm{mm}$ of dental crowding as an inclusion criterion. Many previous researchers have used elastic ring separators or bands as the source of orthodontic force, instead of archwires, for ease of application and under the assumption that they would
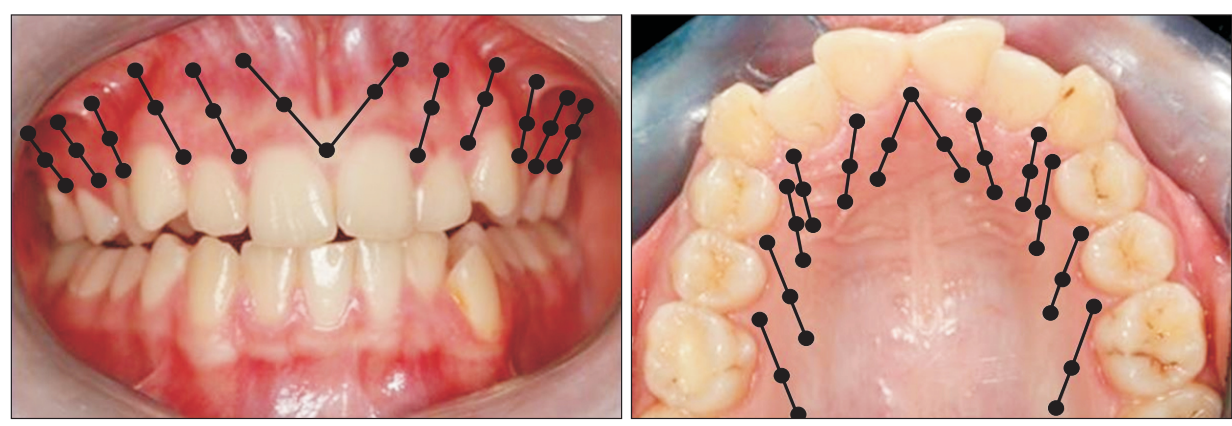

Figure 3. Demonstration of laser applied points. 
Celebi et al • Laser and chewing gum in pain

Table 1. Comparison of age and algometer score between the groups

\begin{tabular}{clcc}
\hline Variable & Group & Mean \pm standard deviation & p-value \\
\hline Age (yr) & Control & $15.3 \pm 1.7$ & $0.587^{*}$ \\
& Laser & $15.4 \pm 1.9$ & \\
\multirow{2}{*}{ Algometer score } & Chewing gum & $15.8 \pm 2.2$ & $0.057^{\dagger}$ \\
& Control & $15.3 \pm 5.4$ & \\
& Laser & $13.9 \pm 3.7$ & \\
& Chewing gum & $17.4 \pm 2.9$ & \\
\hline
\end{tabular}

*Evaluated by one-way ANOVA.

${ }^{\dagger}$ Evaluated by Kruskal-Wallis test.

Table 2. Comparison of visual analog scale score between the groups

\begin{tabular}{|c|c|c|c|}
\hline Time & Group & Mean \pm standard deviation & $p$-value \\
\hline \multirow[t]{3}{*}{ 2nd hour } & Control & $1.49 \pm 1.68$ & 0.465 \\
\hline & Laser & $1.73 \pm 2.09$ & \\
\hline & Chewing gum & $0.94 \pm 1.01$ & \\
\hline \multirow[t]{3}{*}{ 6th hour } & Control & $3.80 \pm 2.72$ & 0.610 \\
\hline & Laser & $3.43 \pm 2.22$ & \\
\hline & Chewing gum & $2.70 \pm 1.92$ & \\
\hline \multirow[t]{3}{*}{ 1st day } & Control & $5.34 \pm 2.35$ & 0.786 \\
\hline & Laser & $4.80 \pm 2.12$ & \\
\hline & Chewing gum & $5.16 \pm 2.08$ & \\
\hline \multirow[t]{3}{*}{ 2nd day } & Control & $4.48 \pm 2.46$ & 0.833 \\
\hline & Laser & $4.20 \pm 2.37$ & \\
\hline & Chewing gum & $4.27 \pm 1.93$ & \\
\hline \multirow[t]{3}{*}{ 3rd day } & Control & $3.55 \pm 2.32$ & 0.757 \\
\hline & Laser & $3.18 \pm 2.04$ & \\
\hline & Chewing gum & $3.07 \pm 1.83$ & \\
\hline \multirow[t]{3}{*}{ 7th day } & Control & $1.18 \pm 1.63$ & 0.774 \\
\hline & Laser & $1.02 \pm 1.19$ & \\
\hline & Chewing gum & $1.42 \pm 1.41$ & \\
\hline
\end{tabular}

*Evaluated by Kruskal-Wallis test.

provide a more constant force. ${ }^{4,9,15}$ We presume that the nature of pain caused by the separator may be different from the pain caused by the archwire. Separators exert only lateral forces for teeth separation, whereas much more complex movements like extrusion, intrusion, and uncontrolled tipping simultaneously occur during the fixed orthodontic treatment using an archwire. Accordingly, archwire placement is clinically more relevant and suitable than separators for the purpose of the study. In addition, when uncontrolled force is applied, separator placement can cause soft tissue injury, thereby becoming a source of pain. Therefore, although it is easier, we did not use separators in our study. It was also ensured that there were no statistically significant differences among the groups in terms of pain threshold.

In this study, neither laser application nor chewing gum was found to be effective for orthodontic pain. Previous studies reported varying results, especially for LLLT. $^{9,15,16}$ There have been reports that laser application is effective in alleviating orthodontic pain; ;5,16 however, contrasting outcomes have also been reported. ${ }^{9}$ Contradictory results in the literature may be due to several reasons. First, LLLT may not genuinely relieve orthodontic pain. Second, results may have been influenced by the variations in technical specifications such as wavelength, power output, energy dose, exposure duration, 


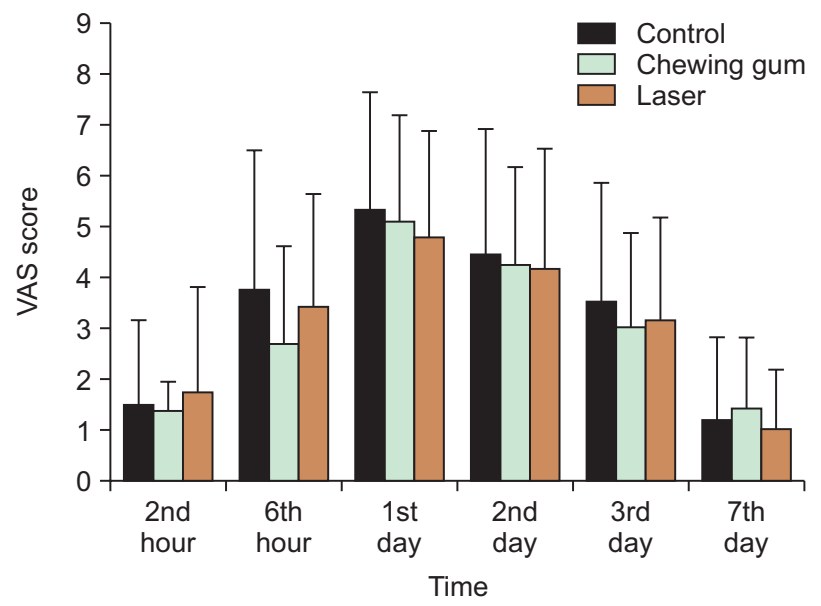

Figure 4. Graphical representation of pain levels in the three groups according to time.

VAS, visual analog scale.

focal spot area, power density, and energy density of the lasers used in the studies. Even if all other features are optimal, the impropriety of a single feature could negatively impact the effectiveness of the laser. Large number of parameters makes it difficult to provide optimal conditions for the best results. In this study, the specifications that yielded positive results in previous studies were conformed to; however, LLLT was found to be ineffective.

We applied single-dose LLLT immediately after initiating the fixed orthodontic treatment. The question arises why the researchers preferred a single dose application. There are many studies that have applied a single dose laser and reported positive results. ${ }^{9,15,21}$ Therefore, we chose single-dose application and found that the laser did not relieve orthodontic pain. Different outcomes may be possible using multiple applications.

According to our results, chewing gum does not relieve orthodontic pain. Proffit suggested that orthodontic pain could be reduced by chewing gum or wafers during the first few hours of appliance activation. ${ }^{22}$ This hypothesis was based on the idea that chewing compressible materials, such as gum or silicon wafers temporarily displaces the teeth. This loosens the compressed periodontal structures, including the nerve fibers and occluded blood vessels, enabling the blood to flow more easily, thereby preventing the accumulation of metabolic products such as algogens that cause hyperalgesic response in the periodontium. ${ }^{1,22,23}$ Although several articles confirmed the accuracy of this hypothesis and presented positive results, ${ }^{17,24}$ some studies have reported contradictory outcomes. ${ }^{18,25}$ Farzanegan et al. ${ }^{24}$ concluded that both chewing gum and viscoelastic bite wafers were effective in pain alleviation, and that these methods could be suggested as suitable substitutes for ibuprofen. However, Alqareer et al. ${ }^{18}$ stated that chewing gum three times a day was not effective in reducing orthodontic pain, when compared to a placebo, recommending instead that clinicians should manage patients' pain expectations. We assume these different outcomes to be due to (1) differences in the methodology of the studies, (2) the fact that pain is influenced by individual variations, and (3) the difficulty of measuring pain. Individual variations were ignored when designing many study. For instance, Benson et al. ${ }^{17}$ did not ensure equal distribution of the sexes among their research groups. They included 9 female and 19 male participants in their non-chewing gum group, and 17 female and 12 male participants in their chewing gum group. 0'Connor ${ }^{6}$ stated that women are more fragile and sensitive to pain, while men are more stoic and more tolerant to pain.

Although it is claimed that compressible materials, such as chewing gum and wafers, reduce orthodontic pain, ${ }^{22}$ chewing these materials may not lead to enough temporary displacement of the teeth to allow blood to flow through compressed areas to prevent a build-up of metabolic products, because the chewing motion is usually performed in a limited direction. There is not much difference between chewing these materials and chewing regular food. The anatomy of the teeth is quite complex, and it seems difficult to rectify the occluded vessels and nerves in the periodontium using this method. Instead of moving the teeth in limited planes, other methods such as vibration devices that offer more complex movements might be useful. However, these devices are expensive, and there are conflicting results in the literature regarding their effectiveness. ${ }^{26-28}$ Future studies are needed to precisely determine the cost-benefit ratio of these devices.

The number of participants can be considered a major limitation of this study. Although 19 participants were found sufficient per group according to power analysis and previous studies conducted with a similar number of participants, ${ }^{15,16}$ we would have preferred to include more participants. Though there was no statistically significant difference among the groups in pain sensitivity, the $p$-value ( $p=0.057$ ) was too close to the significance level, and because of the sample size we had to use non-parametric statistic with which it is harder to observe statistical significance. If the sample size had been large enough to show a normal distribution, parametric statistic, which is more powerful in demonstrating statistical significance, could have been used. There are previous studies with results contradictory to the outcomes of this study, especially regarding LLLT effects. ${ }^{15,16,21}$ Therefore, this limitation should be considered while evaluating our findings.

At the second hour of treatment, low levels of pain 
were detected in almost all participants. The pain gradually increased and peaked at 24-48 hours, after which, a downward trend started, and the pain decreased to very low levels around the seventh day. These findings are consistent with existing literature. Many studies have shown that highest levels of pain were experienced between 24 and 48 hours after the orthodontic appliance was activated, and it reduced to tolerable levels after the third day. ${ }^{1,29,30}$ Chewing gum and laser application did not change this general pattern.

\section{CONCLUSION}

The results of this study revealed that both chewing gum and low-level laser application with the protocol and technical specifications used in the study, have no alleviating effects on orthodontic pain. Future studies, with different protocols and specifications, are needed to evaluate their usefulness.

\section{CONFLICTS OF INTEREST}

No potential conflict of interest relevant to this article was reported.

\section{REFERENCES}

1. Krishnan V. Orthodontic pain: from causes to management--a review. Eur J Orthod 2007;29:170-9.

2. Erdinç AM, Dinçer B. Perception of pain during orthodontic treatment with fixed appliances. Eur J Orthod 2004;26:79-85.

3. Bergius M, Kiliaridis S, Berggren U. Pain in orthodontics. A review and discussion of the literature. J Orofac Orthop 2000;61:125-37.

4. Bergius M, Berggren U, Kiliaridis S. Experience of pain during an orthodontic procedure. Eur J Oral Sci 2002;110:92-8.

5. Lew KK. Attitudes and perceptions of adults towards orthodontic treatment in an Asian community. Community Dent Oral Epidemiol 1993;21:31-5.

6. O'Connor PJ. Patients' perceptions before, during, and after orthodontic treatment. J Clin Orthod 2000;34:591-2.

7. Maquet D, Croisier JL, Demoulin C, Crielaard JM. Pressure pain thresholds of tender point sites in patients with fibromyalgia and in healthy controls. Eur J Pain 2004;8:111-7.

8. Park G, Kim CW, Park SB, Kim MJ, Jang SH. Reliability and usefulness of the pressure pain threshold measurement in patients with myofascial pain. Ann Rehabil Med 2011;35:412-7.

9. Lim HM, Lew KK, Tay DK. A clinical investigation of the efficacy of low level laser therapy in reduc- ing orthodontic postadjustment pain. Am J Orthod Dentofacial Orthop 1995;108:614-22.

10. Schultz RJ, Krishnamurthy S, Thelmo W, Rodriguez JE, Harvey G. Effects of varying intensities of laser energy on articular cartilage: a preliminary study. Lasers Surg Med 1985;5:577-88.

11. Balboni GC, Brandi ML, Zonefrati R, Repice F. Effects of He-Ne/l. R. laser irradiation on two lines of normal human fibroblasts in vitro. Arch Ital Anat Embriol 1986;91:179-88.

12. Harris DM. Editorial comment biomolecular mechanisms of laser biostimulation. J Clin Laser Med Surg 1991;9:277-80.

13. Mester E, Mester AF, Mester A. The biomedical effects of laser application. Lasers Surg Med 1985; 5:31-9.

14. Shirazi M, Ahmad Akhoundi MS, Javadi E, Kamali A, Motahhari P, Rashidpour M, et al. The effects of diode laser $(660 \mathrm{~nm})$ on the rate of tooth movements: an animal study. Lasers Med Sci 2015;30:713-8.

15. Bicakci AA, Kocoglu-Altan B, Toker H, Mutaf 1, Sumer Z. Efficiency of low-level laser therapy in reducing pain induced by orthodontic forces. Photomed Laser Surg 2012;30:460-5.

16. Doshi-Mehta G, Bhad-Patil WA. Efficacy of lowintensity laser therapy in reducing treatment time and orthodontic pain: a clinical investigation. Am J Orthod Dentofacial Orthop 2012;141:289-97.

17. Benson PE, Razi RM, Al-Bloushi RJ. The effect of chewing gum on the impact, pain and breakages associated with fixed orthodontic appliances: a randomized clinical trial. Orthod Craniofac Res 2012; 15:178-87.

18. Alqareer A, Alyahya A, Al-Anezi SA, AlAwadhi A, Al Qabandi S, Alyaseen M. Efficacy of chewing gum to reduce orthodontic pain compared to placebo: a blinded, parallel-group, preliminary clinical trial. J Oral Facial Pain Headache 2019;33:301-7.

19. Kinser AM, Sands WA, Stone MH. Reliability and validity of a pressure algometer. J Strength Cond Res 2009;23:312-4.

20. Jayaseelan DJ, Cole KR, Courtney CA. Hand-held dynamometer to measure pressure pain thresholds: a double-blinded reliability and validity study. Musculoskelet Sci Pract 2021;51:102268.

21. Matys J, Jaszczak E, Flieger R, Kostrzewska-Kaminiarz K, Grzech-Leśniak K, Dominiak M. Effect of ozone and diode laser $(635 \mathrm{~nm})$ in reducing orthodontic pain in the maxillary arch-a randomized clinical controlled trial. Lasers Med Sci 2020;35:48796.

22. Proffit WR. Contemporary orthodontics. 3rd ed. St. Louis: Mosby; 2000.

23. Celebi F, Turk T, Bicakci AA. Effects of low-level la- 
ser therapy and mechanical vibration on orthodontic pain caused by initial archwire. Am J Orthod Dentofacial Orthop 2019;156:87-93.

24. Farzanegan F, Zebarjad SM, Alizadeh S, Ahrari F. Pain reduction after initial archwire placement in orthodontic patients: a randomized clinical trial. Am J Orthod Dentofacial Orthop 2012;141:169-73.

25. Ireland AJ, Ellis P, Jordan A, Bradley R, Ewings P, Atack NE, et al. Comparative assessment of chewing gum and ibuprofen in the management of orthodontic pain with fixed appliances: a pragmatic multicenter randomized controlled trial. Am J Orthod Dentofacial Orthop 2016;150:220-7.

26. Miles P, Smith H, Weyant R, Rinchuse DJ. The effects of a vibrational appliance on tooth movement and patient discomfort: a prospective randomised clinical trial. Aust Orthod J 2012;28:213-8.

27. Lobre WD, Callegari BJ, Gardner G, Marsh CM, Bush AC, Dunn WJ. Pain control in orthodontics using a micropulse vibration device: a randomized clinical trial. Angle Orthod 2016;86:625-30.

28. Marie SS, Powers M, Sheridan JJ. Vibratory stimulation as a method of reducing pain after orthodontic appliance adjustment. J Clin Orthod 2003;37:2058; quiz 203-4.

29. Ngan P, Kess B, Wilson S. Perception of discomfort by patients undergoing orthodontic treatment. Am J Orthod Dentofacial Orthop 1989;96:47-53.

30. Scheurer PA, Firestone AR, Bürgin WB. Perception of pain as a result of orthodontic treatment with fixed appliances. Eur J Orthod 1996;18:349-57. 